\title{
De novo BK channel variant causes epilepsy by affecting voltage gating but not $\mathrm{Ca}^{2+}$ sensitivity
}

\author{
Xia $\mathrm{Li}^{1} \cdot$ Sibylle Poschmann ${ }^{2} \cdot$ Qiuyun Chen ${ }^{3,4} \cdot$ Walid Fazeli $^{5} \cdot$ Nelly Jouayed Oundjian ${ }^{6}$. \\ Francesca M. Snoeijen-Schouwenaars ${ }^{7}$. Oliver Fricke ${ }^{8}$ - Erik-Jan Kamsteeg ${ }^{9} \cdot$ Marjolein Willemsen $^{9,10}$. \\ Qing Kenneth Wang ${ }^{1,3,4}$
}

Received: 18 August 2017 / Revised: 6 November 2017 / Accepted: 23 November 2017 / Published online: 12 January 2018

(c) European Society of Human Genetics 2018

\begin{abstract}
Epilepsy is one of the most common neurological diseases and it causes profound morbidity and mortality. We identified the first de novo variant in KCNMA1 (c.2984 A > G (p.(N995S)))—encoding the BK channel-that causes epilepsy, but not paroxysmal dyskinesia, in two independent families. The c.2984 A $>$ G (p.(N995S)) variant markedly increased the macroscopic potassium current by increasing both the channel open probability and channel open dwell time. The c.2984 A $>\mathrm{G}$ (p.(N995S)) variant did not affect the calcium sensitivity of the channel. We also identified three other variants of unknown significance (c.1554 G > T (p.(K518N)), c.1967A > C (p.(E656A)), and c.3476 A > G (p.(N1159S))) in three separate patients with divergent epileptic phenotypes. However, these variants did not affect the BK potassium current, and are therefore unlikely to be disease-causing. These results demonstrate that BK channel variants can cause epilepsy without paroxysmal dyskinesia. The underlying molecular mechanism can be increased activation of the BK channel by increased sensitivity to the voltage-dependent activation without affecting the sensitivity to the calcium-dependent activation. Our data suggest that the BK channel may represent a drug target for the treatment of epilepsy. Our data highlight the importance of functional electrophysiological studies of BK channel variants in distinguishing whether a genomic variant of unknown significance is a disease-causing variant or a benign variant.
\end{abstract}

\section{Introduction}

Genetic factors significantly contribute to the development of epilepsies, and $>30 \%$ of all epileptic syndromes are considered to be of genetic origin [1]. The next-generation sequencing technology has expedited identification of new

Xia Li, Sibylle Poschmann, Qiuyun Chen, and Walid Fazeli contributed equally to this work.

Electronic supplementary material The online version of this article (https://doi.org/10.1038/s41431-017-0073-3) contains supplementary material, which is available to authorized users.

Publisher's note: Springer Nature remains neutral with regard to jurisdictional claims in published maps and institutional affiliations.

Marjolein Willemsen

marjolein.willemsen@ radboudumc.nl

$\triangle$ Qing Kenneth Wang

wangq2@ccf.org

qkwang@hust.edu.cn

Extended author information available on the last page of the article genes for epilepsies. These and previous genetic studies have resulted in development of several genetic tests that serve diagnostic purposes [1, 2]. However, variants in known genes may be responsible for only $30-50 \%$ of genetic epilepsies $[1,2]$, whereas many additional genes potentially causing epilepsy remain undiscovered.

The KCNMAl gene encodes the large conductance voltage-activated and $\mathrm{Ca}^{2+}$-activated potassium channel $\alpha$ subunit (also referred to as BK channel, calcium-activated potassium channel subunit $\alpha 1$, MaxiK channel, $\mathrm{KCa} 1.1$, or Slo1). We previously reported a functional variant (c.1301 A $>$ G (p.(D434G))) in the BK channel that causes a syndrome of combined generalized epilepsy and paroxysmal dyskinesia (GEPD) by markedly increasing the macroscopic potassium current and enhancing calcium sensitivity of the channel [3]. In this study, we identified a novel de novo variant (c.2984 A > G (p.(N995S))) in the BK channel that causes sole epilepsy in two independent families by employing a distinctly different mechanism from the c.1301 A > G (p.(D434G)) variant causing paroxysmal dyskinesia in addition to epilepsy. Our studies suggest that 
KCNMAl should be considered as a new gene for epilepsy and thus be included into genetic epilepsy panels for genetic testing.

\section{Materials and methods}

\section{Patients}

This study involves five patients/families who were affected with epilepsy and had missense genomic variants identified in the KCNMAl gene and reported to them. KCNMAl variants were identified by whole-exome sequencing and targeted sequencing as previously described [4-6]. The patients sought genetic consultation from their genetic counselors or physicians, who in turn communicated with us for the functionality of KCNMAI variants. Between January 2015 and April 2017, our laboratory received five referrals for consultation of the functionality of four variants identified in the KCNMAl gene among the five independent patients with epilepsy. The variants and related phenotypic data can be found in the LOVD/shared database (www. LOVD.n1/KCNMA1, individual IDs 00132051-00132055). This study was approved by the Institutional Review Boards (IRB) on Human Subject Research at the Cleveland Clinic and the Ethics Committee on Human Subjects at Huazhong University of Science and Technology and other local IRBs. Written informed consent was obtained from the study subjects according to the IRB policies.

\section{Plasmids and mutagenesis}

The human KCNMA1 cDNA (GenBank accession number U23767) and KCNMB4 cDNA (GenBank accession number AF207992) were cloned into a mammalian expression vector pcDNA3.1, resulting in expression constructs KCNMA1-pcDNA3.1 and KCNMB4-pcDNA3.1 for the $\alpha$ and $\beta 4$-subunits of the $B K$ channel, respectively.

Mutations were generated in KCNMAl-pcDNA3.1 using PCR-based site-directed mutagenesis and verified by direct DNA sequence analysis as described by us previously $[3,7]$.

\section{Cell culture and transfection}

HEK293 cells were cultured in 24-well plates with DMEM supplemented with $10 \%$ fetal bovine serum at $37^{\circ} \mathrm{C}$ in an incubator with $5 \% \mathrm{CO}_{2}$ as previously reported [7-9]. These cells were co-transfected with a KCNMAl expression construct and the pEGFP-N1 plasmid using Lipofectamine 2000 (Invitrogen) [7-9]. The transfected cells with a green EGFP signal were then selected for patch clamp recordings.

\section{Patch clamp recording}

Patch clamp recordings were carried out $6-24 \mathrm{~h}$ after the transfection as described by us and others [3, 7-9]. For single-channel recordings, the patches that contain only one channel were selected, and the recordings were digitized at $50 \mathrm{kHz}$ and low-pass-filtered at $5 \mathrm{kHz}$ [3, 8-10]. During the recording, solutions with different concentrations of calcium were applied onto the membrane patches via a perfusion pipette containing eight solution channels. The experiments were performed at room temperatures $\left(22-25^{\circ}\right.$ C). The data were collected from five to nine cells as described for patch-clamping of BK channels $(n=5-9)$ [8, 11]. The solutions used for patch clamp included:

Pipette solution containing (in $\mathrm{mM}$ ): $160 \mathrm{MeSO}_{3} \mathrm{~K}, 2$ $\mathrm{MgCl}_{2}, 10$ HEPES.

Nominal $0 \mu \mathrm{M} \mathrm{Ca}^{2+}$ solution containing (in $\mathrm{mM}$ ): 160 $\mathrm{MeSO}_{3} \mathrm{~K}, 5$ EGTA, 10 HEPES.

$1 \mu \mathrm{M} \mathrm{Ca}^{2+}$ solution containing (in $\mathrm{mM}$ ): $160 \mathrm{MeSO}_{3} \mathrm{~K}, 5$ EGTA, $3.25 \mathrm{CaCl}_{2}, 10$ HEPES.

$10 \mu \mathrm{M} \mathrm{Ca}^{2+}$ solution containing (in $\mathrm{mM}$ ): $160 \mathrm{MeSO}_{3} \mathrm{~K}$, 5 HEDTA, $2.988 \mathrm{CaCl}_{2}, 10$ HEPES.

Recorded data were analyzed with Clampfit (Axon Instruments) and SigmaPlot (SPSS) software programs $[3,8-10]$.

\section{Statistical analysis}

Two-tailed Student's $t$-test was used for comparing the means of two groups of variables. A $P$ value of 0.05 or less was considered to be statistically significant.

\section{Results}

\section{Identification of an identical de novo variant in KCNMA1 in two independent families with epilepsy}

Mutational analysis of the KCNMAl gene (GenBank accession number NM_002247.3) identified a c.2984 A > G (p.(N995S)) variant in two independent patients with epilepsy. Both parents of the two patients were not affected by epilepsy (Fig. 1a) and did not carry this variant (Fig. 1a). Sequencing data validated the paternal relationship. Therefore, the c.2984 A > G (p.(N995S)) variant occurred de novo in both patients.

The first epileptic patient was from the Netherlands, and presented with neonatal convulsions and a developmental delay. At the age of 9 years, she was diagnosed with primary generalized myoclonic absence epilepsy that was under control with levetiracetam. Seizures occurred only sporadically when the patient was tired. An EEG at the age of 17 years showed multifocal epileptic discharges. Brain 
a

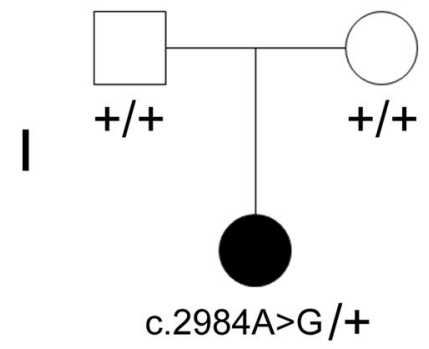

b

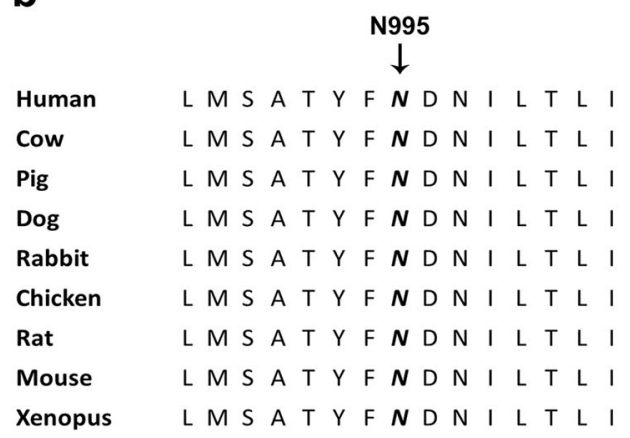

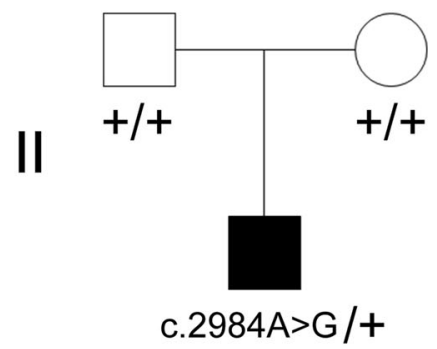

C

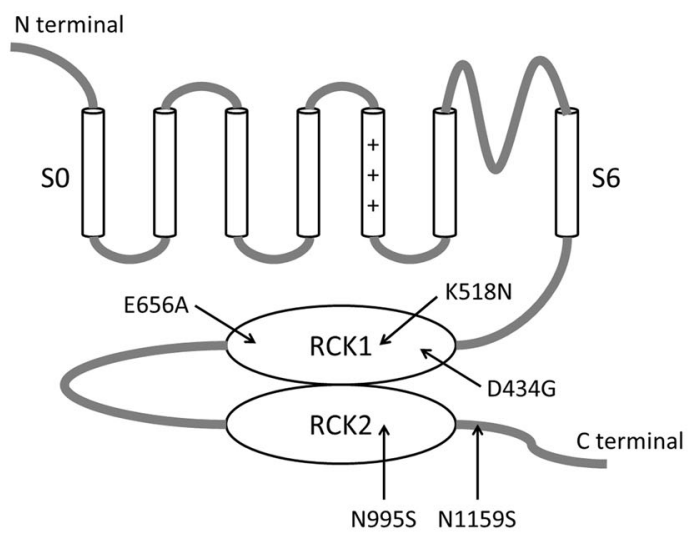

f d

$-180 \mathrm{mV} \frac{120 \mathrm{mV}}{-\frac{\bar{A}^{2}}{2} \mathrm{~m} \mathrm{~m} V--}-120 \mathrm{mV}$
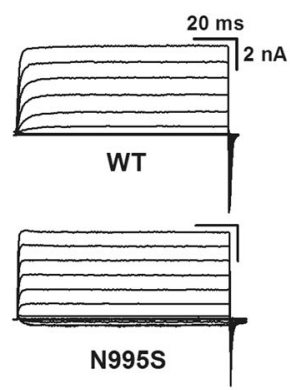

g

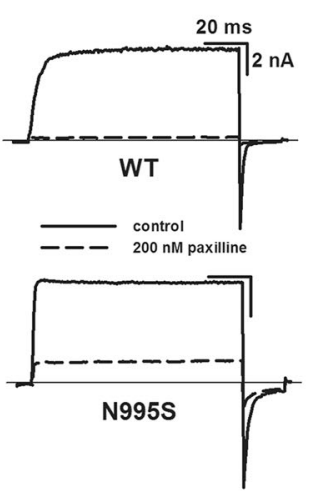

e

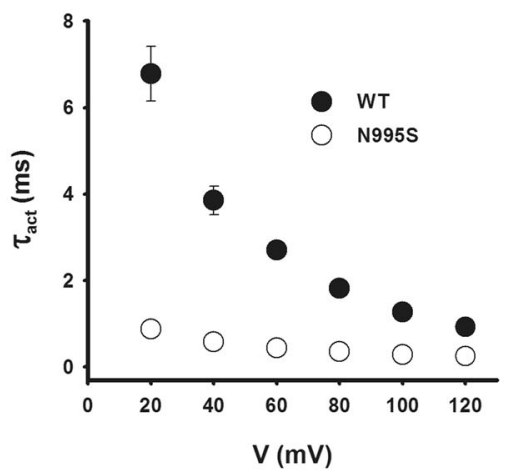

h

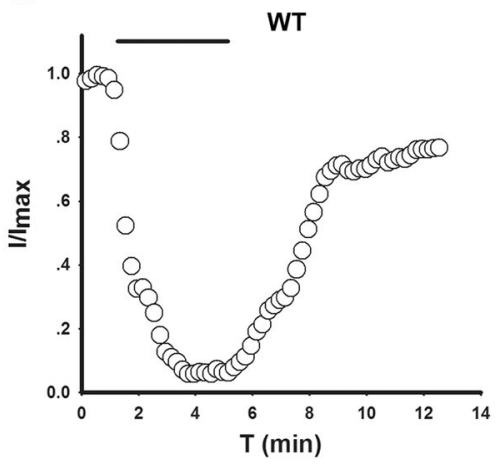

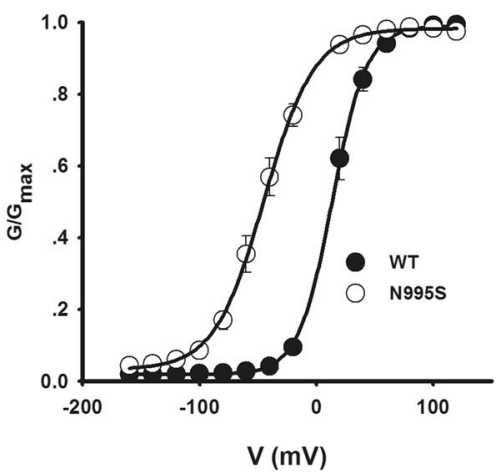

i

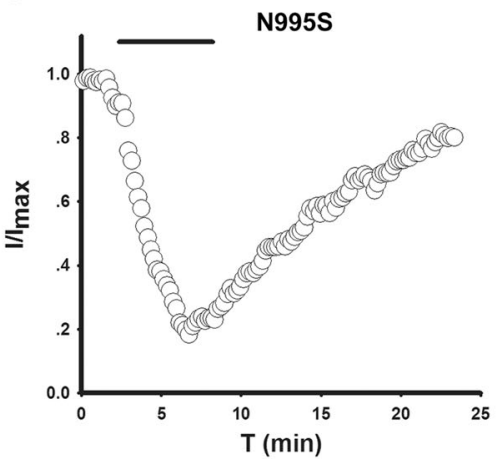

MRI at the age of 17 years showed slight left-sided insular white matter abnormalities. At the age of 23 years, she had a short stature of $155 \mathrm{~cm}$ and small head circumference of
$52.5 \mathrm{~cm}$. She had no evident facial dysmorphic features. Neurological examinations revealed no paroxysmal dyskinesia or other obvious movement disorders. 
Fig. 1 Identification and electrophysiological characterization of a BK channel variant p.(N995S) causing epilepsy. a Pedigrees of the two independent families. Squares, males; circles, females; filled symbol, affected individual; open symbols, unaffected individuals. b The p. (N995) residue, shown in bold and italic, of KCNMA1 is evolutionally conserved among different species. c Structure of the BK channel with the p.(N995S) variant indicated. The p.(D434G) variant identified previously in a large family with generalized epilepsy and paroxysmal dyskinesia, and three other variants identified in this study are also shown. d Representative macroscopic currents of WT and p.(N995S) mutant BK channels from inside-out patches in the presence of $10 \mu \mathrm{M}$ $\mathrm{Ca}^{2+}$ by the protocol as indicated. e Activation time constants of WT and p.(N995S) mutant BK channels at $10 \mu \mathrm{M} \mathrm{Ca}^{2+}$ are plotted against membrane potentials. $\mathbf{f ~ G - V}$ curves of WT and p.(N995S) mutant BK channels at $10 \mu \mathrm{M} \mathrm{Ca}^{2+}$. All $\mathrm{G}-\mathrm{V}$ curves are fitted by Boltzmann function (solid lines) with $V_{1 / 2}(14.1 \pm 6.6 \mathrm{mV}$ for WT and $-44.6 \pm$ $8.2 \mathrm{mV}$ for p.(N995S)). The data are presented as mean $\pm \mathrm{SD}(n=6$, $P<0.05)$. g The representative traces of WT and p.(N995S) mutant BK channels from inside-out patches evoked by a test pulse of $100 \mathrm{mV}$ in the presence of $10 \mu \mathrm{M} \mathrm{Ca}^{2+}$ before (solid) and after (dash) application of $200 \mathrm{nM}$ paxilline. h, i Normalized currents of WT and p. (N995S) mutant BK channels are plotted vs. time. The horizontal bar indicates the treatment duration of $200 \mathrm{nM}$ paxilline

The second patient was from Germany, and showed atypical absence epilepsy beginning at the age of 20 months. Semiology findings included arrest, mouth opening, head turning, variable altered consciousness and occasionally stronger seizures with risk of falls, duration of $10-20 \mathrm{~s}$, and a frequency of $>20$ per day. At the age of 3 years, she developed additional myoclonic seizures, which occurred one to three times per day. No paroxysmal dyskinesia was found. She was a couple of days seizure-free during varicella-zoster and Streptococcus pyogenes infection. Her psychomotor development was mildly delayed. MRI scan and metabolic tests showed normal results. Her EEG showed generalized spike waves located centrally and frontally, and photosensibility. Antiepileptic treatment with ethosuximide led to significant worsening of seizures, and oxcarbazepine and valproate showed negative effects. Sultiame and perampanel did not influence the seizures, either. Zonisamide alone reduced the seizure frequency, but did not stop the myoclonic seizures. Levetiracetam reduced the seizure frequency and stopped myoclonic seizures. She is currently treated with levetiracetam alone.

The c. $2984 \mathrm{~A}>\mathrm{G}$ (p.(N995S)) variant involves an amino-acid residue, which is highly conserved among different species during evolution (Fig. 1b). Moreover, this variant occurs within the second $\mathrm{Ca}^{2+}$-responsive $\mathrm{RCK}$ domain (Fig. 1c). The c.2984 A > G (p.(N995S)) variant does not exist in the public databases (ExAC, GNOMAD, $1 \mathrm{KGP}$, and NHLBI ESP; Table 1). Identification of the same de novo variant in two independent, unrelated families provides the strongest genetic evidence that the c.2984 A >
G (p.(N995S)) variant causes epilepsy shared by the two families.

\section{The p.(N995S) variant markedly increases the macroscopic potassium current of the BK channel}

To determine whether the c.2984 A > G (p.(N995S)) variant has a functional effect on BK channels, we overexpressed the mutant p.(N995S) channel and control wild-type (WT) channel in HEK293 cells, recorded the potassium currents, and analyzed the current-voltage relationship. At an intracellular calcium concentration of $10 \mu \mathrm{M}$, the p.(N995S) variant markedly increased the activation rate (Fig. 1e) and caused a large shift of the $\mathrm{G}-\mathrm{V}$ curve to the negative potentials by $59 \mathrm{mV}(n=6, P<0.05$; Fig. 1f). These data suggest that the p.(N995S) mutant BK channel opens more easily and faster than the WT channel and that the mutant channel generates a greater macroscopic BK current at a given calcium concentration and a membrane potential than its WT counterpart.

Because the above data indicate that the p.(N995S) variant appears to be a gain-of-function variant, which significantly increases the BK current, BK channel inhibitors may block the $\mathrm{BK}$ potassium current and treat epilepsy. We examined the effect of paxilline, a selective BK channel blocker [12], on the potassium currents generated by WT and p.(N995S) mutant BK channels. Treatment with $200 \mathrm{nM}$ of paxilline abolished the current from WT BK channel (Figs. 1g, h, i). While the p.(N995S) mutant BK channels are also sensitive to paxilline, it did not abolish the current (Figs. 1g, h, i).

Because the $\beta 4$ subunit of the BK channel is the subunit predominantly expressed in the central nervous system [13, 14], we studied the effect of the epilepsy-linked p.(N995S) variant on the $\mathrm{BK}$ channel in the presence of the $\beta 4$ subunit and characterized the current-voltage relationship of the WT

Table 1 Frequencies of $K C N M A 1$ variants in public databases

\begin{tabular}{lllll}
\hline & \multicolumn{4}{l}{ Public database } \\
\cline { 2 - 5 } Variant & A & B & C & D \\
\hline c.2984A $>$ G (p.(N995S)) & 0 & 0 & 0 & 0 \\
c.3476A $>$ G (p.(N1159S)) & $6 / 121,392$ & $25 / 277,126$ & $1 / 5008$ & 0 \\
c.1967A $>$ C (p.(E656A)) & 0 & $3 / 245360$ & 0 & 0 \\
c.1554G $>$ T (p.(K518N)) & 0 & $3 / 246230$ & 0 & 0 \\
\hline
\end{tabular}

(A) ExAC Exome Aggregation Consortium (http://exac.broadinstitute. org)

(B) GNOMAD Genome Aggregation Database (http://gnomad.broa dinstitute.org)

(C) $1 K G P 1000$ Genomes Project (http://browser.1000genomes.org)

(D) NHLBI ESP Exome Variant Server (http://evs.gs.washington.edu/ EVS) 
Fig. 2 The $\beta 4$ subunit slightly augments the effect of the p. (N995S) variant on channel activation. a Representative macroscopic currents of WT and p.(N995S) mutant BK channels with $\mathrm{h} \beta 4$ from inside-out patches in the presence of $10 \mu \mathrm{M} \mathrm{Ca}^{2+}$ by the protocol as indicated. b $\mathrm{G}-\mathrm{V}$ curves of $\mathrm{WT}+\mathrm{h} \beta 4$ and p. $(\mathrm{N} 995 \mathrm{~S})+\mathrm{h} \beta 4$ at $10 \mu \mathrm{M} \mathrm{Ca}^{2}$ ${ }^{+}$. All G-V curves are fitted by Boltzmann function (solid lines) with $V_{1 / 2}(24.1 \pm 8.7 \mathrm{mV}$ for $\mathrm{WT}+\mathrm{h} \beta 4$ and $-47.1 \pm 7.9 \mathrm{mV}$ for $p .(\mathrm{N} 995 \mathrm{~S})+\mathrm{h} \beta 4)$. The data are presented as mean $\pm \mathrm{SD}$ $(n=6, P<0.05)$ a

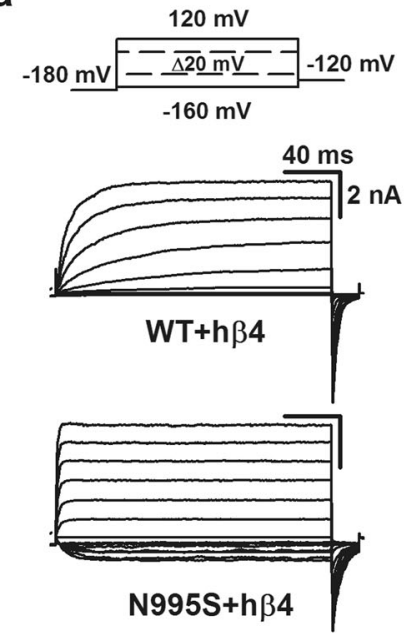

b

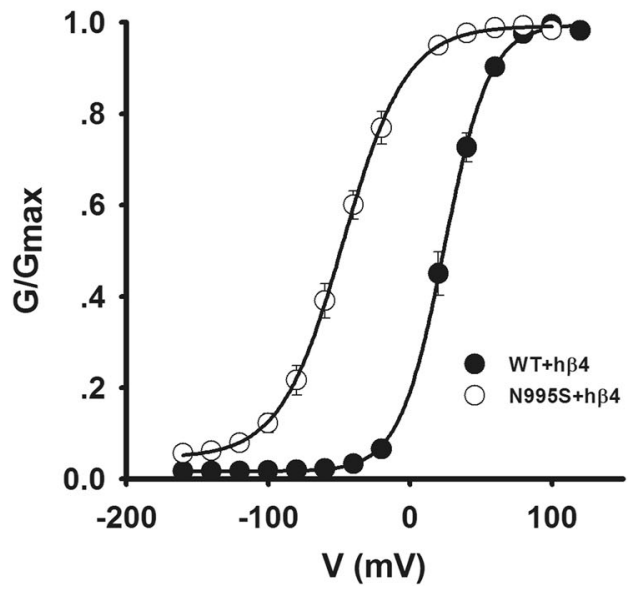

and mutant BK channels in the presence of the $\beta 4$ subunit. The p.(N995S) variant markedly enhanced the activation of BK channels (Fig. 2a) and shifted the G-V curve to a negative potential by $71 \mathrm{mV}(n=6, P<0.05$; Fig. $2 \mathrm{~b}$ ) in the presence of the $\beta 4$ subunit at a calcium concentration of $10 \mu \mathrm{M}$.

\section{The p.(N995S) variant does not affect the calcium sensitivity of the BK channel}

We recorded the currents from WT and mutant BK channels under a series of calcium concentrations, including nominal 0,1 , and $10 \mu \mathrm{M}$ (Fig. 3a). The $V_{1 / 2}$ values differed between the p.(N995S) mutant and WT channels at all three calcium concentrations (Fig. 3b). Similarly, the absolute values of $\Delta V_{1 / 2}\left(V_{1 / 2}\right.$ (p.(N995S)) $\left.-V_{1 / 2}(\mathrm{WT})\right)$ for the p.(N995S) mutant and WT channels were $>40 \mathrm{mV}$ at all three calcium concentrations ( $n=6, P<0.05$; Fig. $3 c)$. However, the differences for the $V_{1 / 2}$ values between the p.(N995S) mutant and WT channels remained similar independent from the calcium concentrations (Fig. 3c), suggesting that the p.(N995S) variant increases BK channel activation without enhancing calcium sensitivity. Moreover, the $\mathrm{p}$. (N995S) mutant channel remained highly activated even at a nominal calcium concentration of $0 \mu \mathrm{M}$ (Figs. 3a, b).

\section{The p.(N995S) variant continues to enhance activation of the channel even when the calcium response of the $B K$ channel is blocked}

We studied the effect of the p.(N995S) variant in BK channels that harbor mutations at two putative calcium-binding sites, which are referred to as p.2D2A (p.(D427A), p. (D432A)), and p.5D5N (p.(D959N), p.(D960N), p.(D961N), p.(D962N), p.(D963N)). The p.(N995S) mutant channels continued to generate greater macroscopic potassium currents compared to WT channels even when the two calcium- binding sites were mutated. Moreover, the G-V curves of the p.(N995S) mutant channel were still shifted to negative potentials by more than $40 \mathrm{mV}$ at nominal calcium concentrations of 0,1 , and $10 \mu \mathrm{M}$ without a change of the slope as compared to the WT channel (Fig. 3d). The differences of the $V_{1 / 2}$ values between the p.(N995S) mutant channels and the WT channels were identical at nominal 0,1 , and $10 \mu \mathrm{M}$ of calcium (Fig. 3e) when the calcium-dependent activation pathway of BK channels was blocked by the p.2D2A/ p.5D5N mutations. Therefore, although the p.(N995S) variant is located within the second RCK domain, its effect is not associated with the calcium sensitivity of the BK channel.

\section{The p.(N995S) variant stabilizes the open state of BK channels}

To further investigate the functional impact of the $\mathrm{p}$. (N995S) variant on BK channels, we analyzed its effects on single-channel properties. Both the WT channel and the p. (N995S) mutant channel were activated by an increase in voltages at a given calcium concentration. The p.(N995S) mutant channels open more frequently than the WT channels (Fig. 4a). The single-channel conductance of the WT and mutant channels were $268.5 \pm 13.2(\mathrm{pS})$ and $276.3 \pm$ 18.9 (pS), respectively (Fig. 4a). There is no statistically significant difference between the WT and mutant channels for the single-channel conductance $(n=9, P>0.05)$, which indicates that the p.(N995S) variant does not affect the pore structure of the BK channel. The $P_{\mathrm{o}}-V$ curve of the $\mathrm{p}$. (N995S) mutant channels shifted to negative potentials at both $1 \mu \mathrm{M} \mathrm{Ca}^{2+}$ and $10 \mu \mathrm{M} \mathrm{Ca}^{2+}$ compared with the WT BK channels (Fig. 4b), which is consistent with the data from the macroscopic current recordings (Fig. 1). The distribution of the open dwell time was different between the WT channels and the mutant channels. The open dwell time histograms were fitted by an exponential function, and the 
a

WT

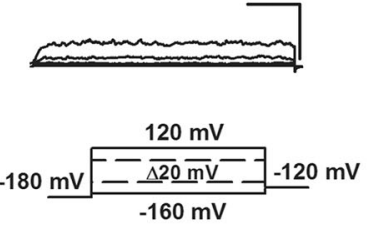

N995S

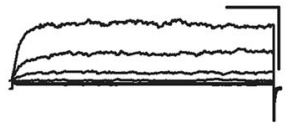

$0 \mu \mathrm{M} \mathrm{Ca}^{2+}$
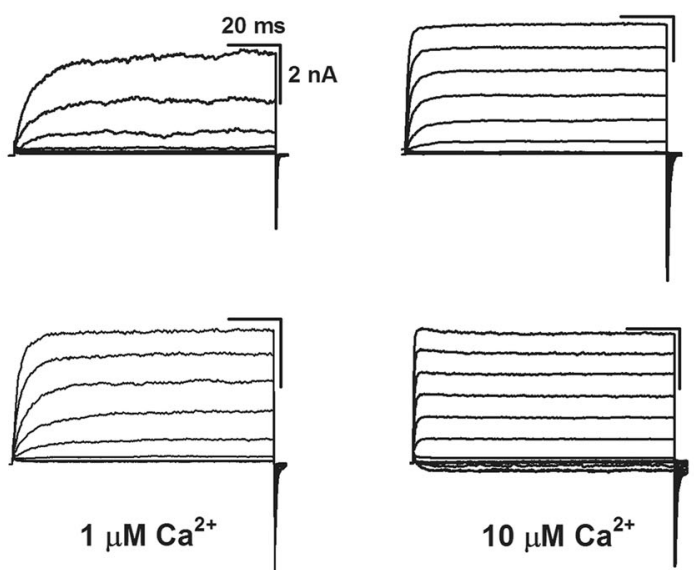

b

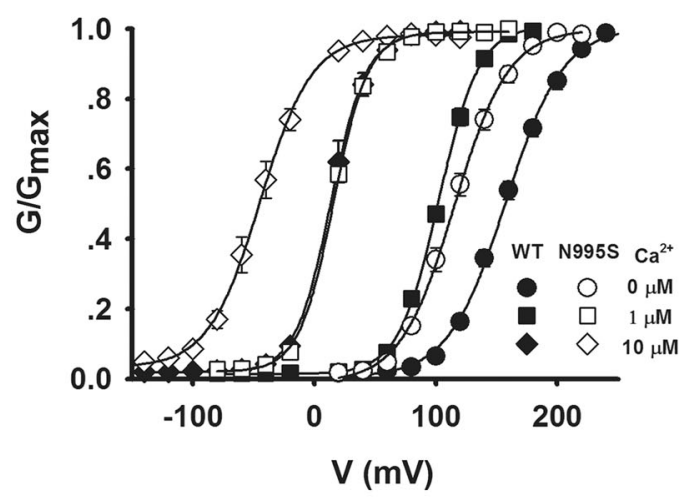

d

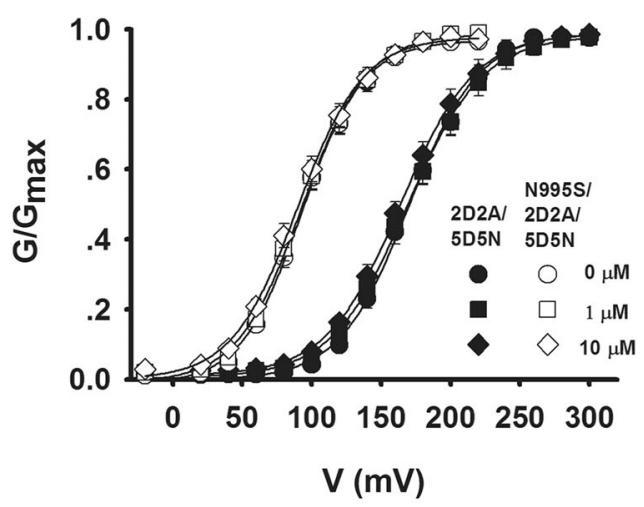

Fig. 3 Variant p.(N995S) has little effect on the $\mathrm{Ca}^{2+}$-dependent activation mechanism of the BK channel. a Representative macroscopic currents of WT (upper panel) and p.(N995S) mutant (lower panel) $\mathrm{BK}$ channels from inside-out patches at nominal $0 \mu \mathrm{M} \mathrm{Ca}^{2+}$ (left), $1 \mu \mathrm{M} \mathrm{Ca}^{2+}$ (middle), and $10 \mu \mathrm{M} \mathrm{Ca}^{2+}$ (right) by the protocol as indicated. b G-V curves of WT and p.(N995S) mutant BK channels at nominal $0 \mu \mathrm{M} \mathrm{Ca}^{2+}, 1 \mu \mathrm{M} \mathrm{Ca}^{2+}$, and $10 \mu \mathrm{M} \mathrm{Ca}^{2+}$. All G-V curves are fitted by Boltzmann function (solid lines) with $V_{1 / 2}$ at nominal $0 \mu \mathrm{M}$ $\mathrm{Ca}^{2+}(158.2 \pm 6.3 \mathrm{mV}$ for WT and $115.8 \pm 6.9 \mathrm{mV}$ for $\mathrm{p}$.(N995S), $P<$ $0.05)$, at $1 \mu \mathrm{M} \mathrm{Ca}^{2+}(102.8 \pm 2.6 \mathrm{mV}$ for WT and $15.8 \pm 3.5 \mathrm{mV}$ for $\mathrm{p}$. (N995S), $P<0.05)$, and at $10 \mu \mathrm{M} \mathrm{Ca}^{2+}(14.1 \pm 6.6 \mathrm{mV}$ for WT and $-44.6 \pm 8.2 \mathrm{mV}$ for p.(N995S), $P<0.05)$. c $V_{1 / 2}$ of $\mathrm{G}-\mathrm{V}$ curves vs. $\left[\mathrm{Ca}^{2+}\right]_{\mathrm{i}}$ for WT and p.(N995S) mutant BK channels. $\mathbf{d} \mathrm{G}-\mathrm{V}$ curves of
C

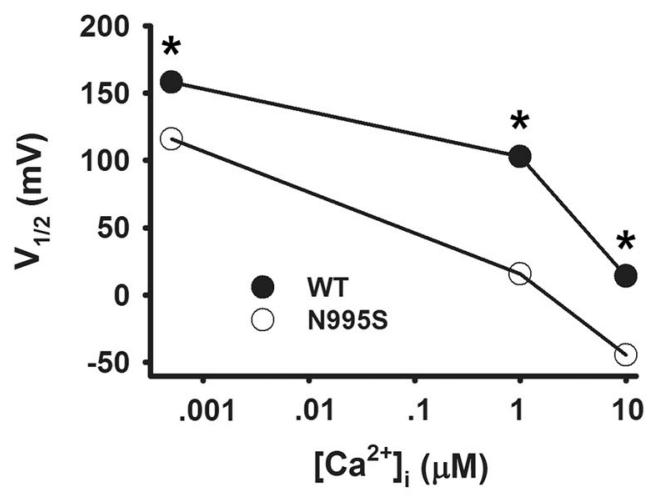

e

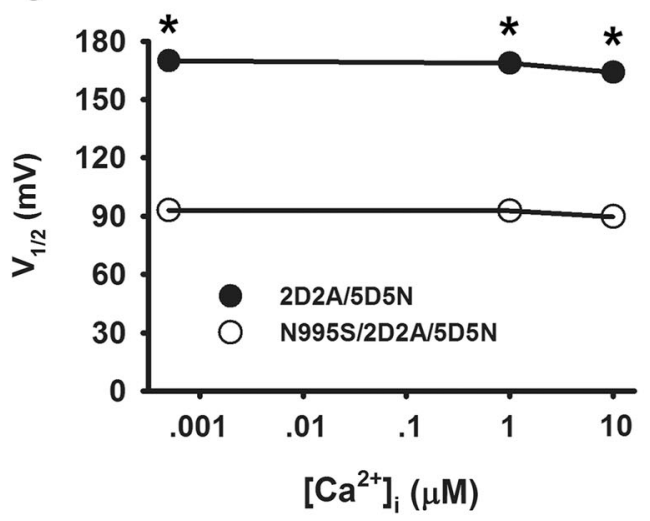

p.2D2A/p.5D5N and p.(N995S)/p.2D2A/p.5D5N mutant BK channels at nominal $0 \mu \mathrm{M} \mathrm{Ca}^{2+}, 1 \mu \mathrm{M} \mathrm{Ca}^{2+}$, and $10 \mu \mathrm{M} \mathrm{Ca}^{2+}$. All G-V curves are fitted by Boltzmann function (solid lines) with $V_{1 / 2}$ and slope factor at nominal $0 \mu \mathrm{M} \mathrm{Ca}^{2+}(169.8 \pm 7.1 \mathrm{mV}, 24.6 \pm 2.9$ for p. $2 \mathrm{D} 2 \mathrm{~A} /$ p.5D5N and $92.8 \pm 4.9 \mathrm{mV}, 22.0 \pm 2.6$ for p. (N995S)/p.2D2A/ p.5D5N, $\left.P_{(V 1 / 2)}<0.05\right)$, at $1 \mu \mathrm{M} \mathrm{Ca}^{2+}(168.6 \pm 6.6 \mathrm{mV}, 26.2 \pm 3.0$ for p.2D2A/p.5D5N and $92.7 \pm 5.1 \mathrm{mV}, 23.2 \pm 2.1$ for p.(N995S)/ p.2D2A/p.5D5N, $\left.P_{(V 1 / 2)}<0.05\right)$ and at $10 \mu \mathrm{M} \mathrm{Ca}^{2+}(164.0 \pm 6.3 \mathrm{mV}$, $25.5 \pm 3.2$ for p. $2 \mathrm{D} 2 \mathrm{~A} / \mathrm{p} .5 \mathrm{D} 5 \mathrm{~N}$ and $89.7 \pm 5.0 \mathrm{mV}, 23.6 \pm 2.5$ for $\mathrm{p}$. (N995S)/p.2D2A/p.5D5N, $\left.P_{(V 1 / 2)}<0.05\right)$. e $V_{1 / 2}$ of $\mathrm{G}-\mathrm{V}$ curves vs. $\left[\mathrm{Ca}^{2+}\right]_{\mathrm{i}}$ for p.2D2A/p.5D5N and p.(N995S)/p.2D2A/p.5D5N mutant BK channels. The data are presented as mean $\pm \mathrm{SD}(n=6, P<0.05)$ 
a

WT
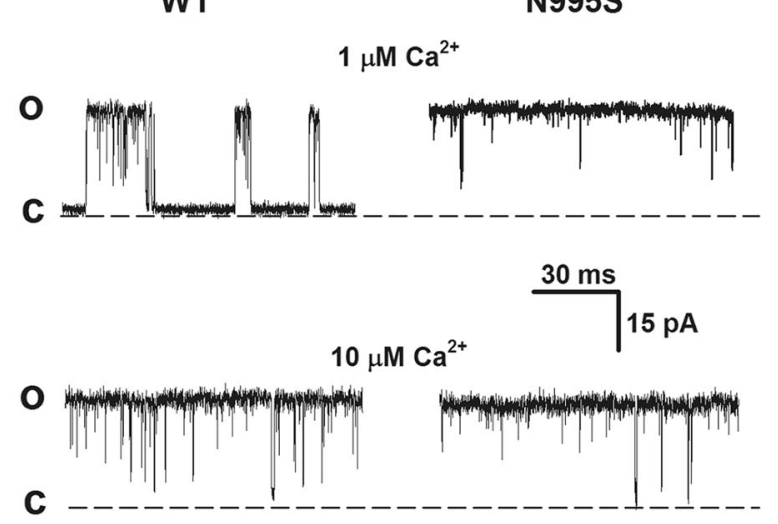

b

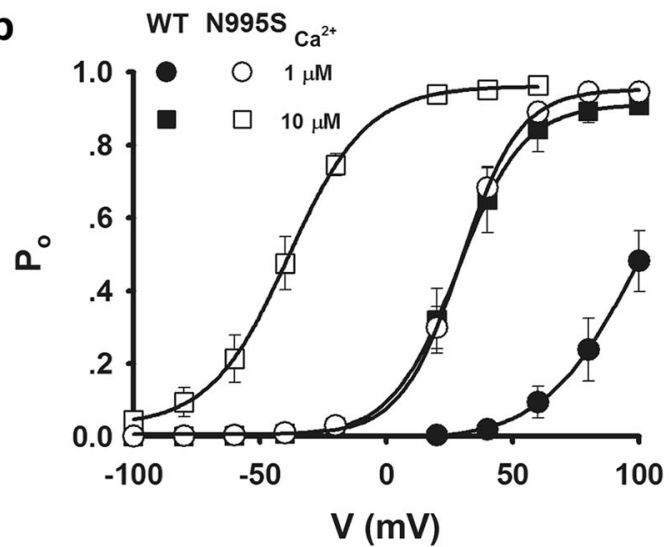

Fig. 4 Single-channel electrophysiological analysis of the p.(N995S) variant of BK channel causing epilepsy. a Representative singlechannel currents of WT and p.(N995S) mutant BK channels at $1 \mu \mathrm{M}$ $\mathrm{Ca}^{2+}$ and $10 \mu \mathrm{M} \mathrm{Ca}^{2+}$ recorded at $100 \mathrm{mV}$. C and $\mathrm{O}$ indicate the closed and open state of the channel, respectively. The sample rate is $50 \mathrm{kHz}$, and the low-pass filter is $5 \mathrm{kHz}$. b Open probability $P_{\mathrm{o}}-V$ curves of WT and p.(N995S) mutant BK channels at $1 \mu \mathrm{M} \mathrm{Ca}^{2+}$ and $10 \mu \mathrm{M} \mathrm{Ca}^{2+}$. $\mathbf{c}$ Representative open dwell time histograms of WT and

time constant of the mutant channels was found to be larger than that of WT channels at the same potentials and calcium concentrations ( $n=5, P<0.05$; Figs. $4 \mathrm{c}, \mathrm{d}$ ). This suggests that the mutant channels remain open for a longer period compared with WT channels. The open state of the mutant channels appears to be more stable than WT channels. Thus, the single-channel analysis identified the mechanism by which the p.(N995S) variant enhances BK macroscopic currents to be the increased single-channel open probability and increased open dwell time.

\section{Identification and characterization of three other genomic variants in KCNMA1}

Whole-exome sequencing analysis identified three other genomic variants in KCNMAl in three other patients with

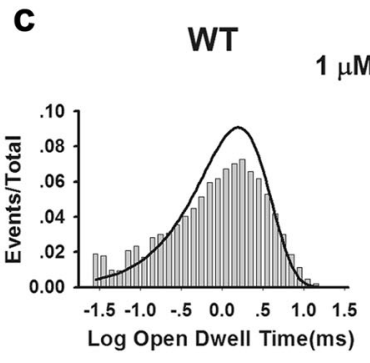

\section{$\mu \mathrm{M} \mathrm{Ca}{ }^{2+}$ \\ N995S}

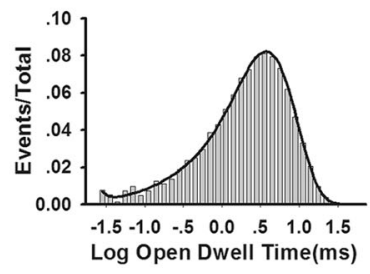

$10 \mu \mathrm{M} \mathrm{Ca}^{2+}$
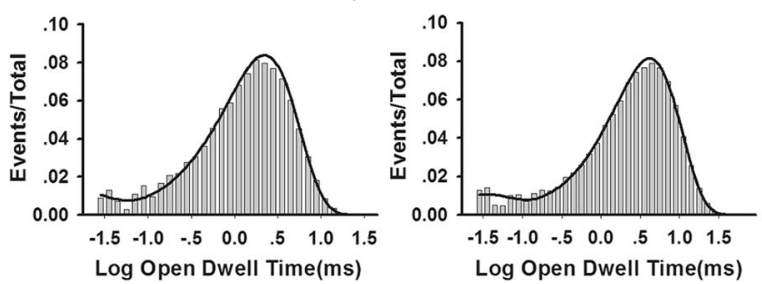

d
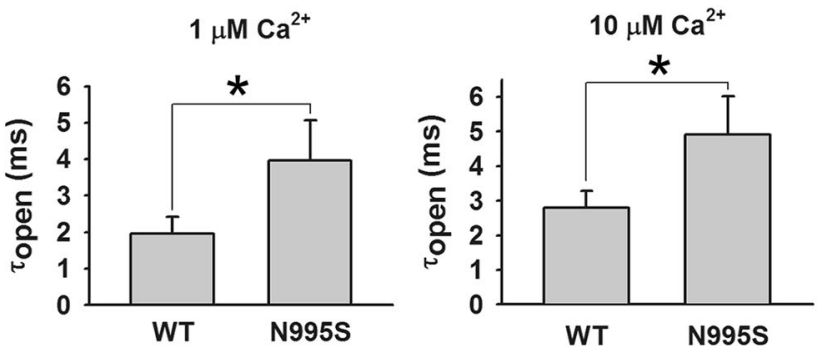

p.(N995S) mutant BK channels at $1 \mu \mathrm{M} \mathrm{Ca}^{2+}$ and $10 \mu \mathrm{M} \mathrm{Ca}^{2+}$ measured at $100 \mathrm{mV}$. Histograms are plotted in log-bin timescales and fitted by exponential function (solid lines). d Time constants of open dwell time for WT and p.(N995S) mutant BK channels at $1 \mu \mathrm{M} \mathrm{Ca}{ }^{2+}$ $(1.9 \pm 0.5 \mathrm{~ms}$ for WT and $3.9 \pm 1.1 \mathrm{~ms}$ for p.(N995S), $P<0.05)$ and at $10 \mu \mathrm{M} \mathrm{Ca}^{2+}(2.8 \pm 0.5 \mathrm{~ms}$ for WT and $4.9 \pm 1.1 \mathrm{~ms}$ for p.(N995S), $P$ $<0.05)$. The data are presented as mean $\pm \mathrm{SD}(n=5, P<0.05)$

epilepsy, i.e., c.3476 A>G (p.(N1159S)) in a Chinese patient with epilepsy (Supplementary Figure S1a-c), c.1967 A > C (p.(E656A)) in a 30-year-old US patient with severe epilepsy (absence, atonic, myoclonic, tonic, and other types; Supplementary Figure S2a and b) and c.1554 G > T (p. $(\mathrm{K} 518 \mathrm{~N})$ ) in a German patient with epileptic encephalopathy (Supplementary Figure S3a and b). The c.3476 A > G (p.(N1159S)) variant was found in the ExAC, GNOMAD, and $1 \mathrm{KGP}$ databases, whereas the other two variants were found in the GNOMAD database (Table 1). The father of the patient with the c.3476 A > G (p.(N1159S)) variant also carried the same variant, but was not affected with epilepsy (Supplementary Figure S1a). The patient with the c.1967A $>$ C (p.(E656A)) variant was adopted and we could not determine whether one of the parents carries the variant. The parents of the patient with the c.1554G>T (p. 
(K518N)) variant did not want to be tested genetically; thus, we could not determine whether the variant occurred de novo. No differences were observed between the three mutant channels and WT channels with regard to the activation and current density of the BK channels in the presence or absence of the $\beta 4$ subunit (Supplementary Figures S1-S3). These data suggest that the three variants are not likely to affect the function of BK channels, and instead may represent benign genetic variants.

\section{Discussion}

Our data in the present study demonstrate that variants in the large conductance calcium-sensitive potassium BK channel can cause epilepsy. This conclusion is strongly supported by both genetic and electrophysiological studies. The identification of one novel de novo variant, c.2984 A > G (p.(N995S)), in the BK channel in two independent patients with epilepsy provides sufficient genetic evidence to demonstrate that c.2984 A > G (p.(N995S)) can be causative to epilepsy. Electrophysiological characterization of the p.(N995S) mutant BK channel demonstrated that the variant is a gain-of-function variant that markedly affects the functional properties of the BK channel.

We have identified a novel molecular mechanism by which a variant in the KCNMAl gene can cause epilepsy. The p.(N995S) variant leads to a significant increase of the macroscopic potassium current of the BK channel by shortening the channel activation time and markedly shifting the $\mathrm{G}-\mathrm{V}$ curve to negative potentials by $59 \mathrm{mV}$ at 10 $\mu \mathrm{M} \mathrm{Ca}^{2+}$ (Fig. 1). The p.(N995S) variant can enhance the BK current by increasing the open probability and significantly prolonging the channel open dwell time without enhancing calcium sensitivity (Figs. 2 and 3).

The c.1301 A > G (p.(D434G)) variant in KCNMA1 causes a phenotype characterized by the co-existence of GEPD [3], whereas the c.2984 A>G (p.(N995S)) variant in this study causes epilepsy only. We found that the functional effects of the p.(D434G) and p.(N995S) variants are distinctly different. The p.(D434G) variant affects both the voltage activation pathway and the calcium-dependent activation pathway; however, the p.(N995S) variant does not affect the calcium sensitivity. These data suggest that the calcium-activation pathway of the BK channel may be associated with paroxysmal dyskinesia, but not with epilepsy. However, the underlying molecular mechanism is not clear. We have shown that the changes of intracellular calcium concentrations have significant effects on mutant BK channels with the p.(D434G) variant associated with paroxysmal dyskinesia [3]. The release of calcium ions out of the sarcoplasmic reticulum that occurs during contraction of muscles may affect the activity of mutant BK channels with the p.(D434G) variant, potentially leading to paroxysmal dyskinesia. However, mutant BK channels with the p. (N995S) variant are insensitive to the changes of intracellular calcium concentrations during contraction of muscles and, therefore, do not increase the risk of paroxysmal dyskinesia.

It is interesting to note that there are phenotypic differences between the two female patients carrying the same c. $2984 \mathrm{~A}>\mathrm{G}$ (p.(N995S)) variant. The age of onset was 9 years for the patient from the Netherlands, but 20 months for the patient from Germany. The epilepsy for the patient from the Netherlands was primary generalized myoclonic absence epilepsy, but was atypical absence epilepsy for the patient from Germany. The difficulties in assessing genotype-phenotype correlations in genetic epilepsies is well known, and unfortunately poorly understood. For example, the identical variant in $S C N 2 A$ encoding for the voltage-gated sodium channel $\mathrm{Na}_{\mathrm{V}} 1.2$ was associated with a broad spectrum of epilepsies [15]. Recently, a large-scale comparison between two $C$. elegans isolates suggested that differences in the manifestation of mutant phenotypes between individuals are largely the result of natural variation in gene expression [16]. In our cases, potential mechanisms underlying the different phenotypes between the two patients carrying the same c.2984 A>G (p. (N995S)) variant may be related to genetic modifiers (i.e., variants in other genes), environmental/exogenous factors, and/or natural variation in gene expression.

Of note, levetiracetam was effective against epileptic seizures in both patients with the c.2984 A > G (p.(N995S)) variant. Levetiracetam was shown to inhibit presynaptic calcium channels [17-19], but our data suggest that the c.2984 A > G (p.(N995S)) variant causes epilepsy without affecting the calcium-activation pathway of BK channels. Thus, the underlying mechanism for the efficacy of levetiracetam is an interesting topic to be further investigated in the future. Moreover, because we found that the increased BK current is a cause of epilepsy, BK channel inhibitors or blockers may be a novel strategy for the treatment of epilepsy. In line with this, intraperitoneal injection of the BK channel blocker paxilline eliminated tonic-clonic seizures and reduced seizure duration and intensity, which were induced in rats by injection of the gamma-aminobutyric acid (A) antagonists picrotoxin or pentylenetetrazole [20]. We found that treatment with $200 \mathrm{nM}$ paxilline markedly reduced the potassium currents from both WT BK channels and p.(N995S) mutant BK channels (Figs. 1g, h, i). Some residual potassium current was still present in p.(N995S) mutant BK channels at the specific paxilline concentration (Figs. 1g, h, i), which may be beneficial because a complete abolition of the BK current is too toxic for treatment. It is possible to identify a dose of paxilline that may block the BK current from mutant channels to a physiological level, 
which may be used to treat epilepsy. Future effort can also target the BK channel to develop specific inhibitors or blockers of the mutant BK channel activity suitable for the treatment of human patients with epilepsy.

The molecular mechanism by which variant c.2984 A > G (p.(N995S)) affects the function of the BK channel remains to be identified. On the basis of the crystal structure of the cytoplasmic domain of the BK channel [21, 22], the p.N995 residue is located within the "turn" in the helix-turnhelix connector for the "flexible interface" that connects the RCK1 and RCK2 domains. The amino-acid residues in the helix are important for the calcium-dependent activation of the BK channel [21, 22]. The p.N995 residue is in the turn of the helix-turn-helix connector (i.e., not in the helix); thus, it is not involved in the calcium-dependent activation of the BK channel. On the other hand, the turn of the helix-turnhelix connector may be important for the overall molecular conformation of the BK channel through strengthening the interaction between the two RCK domains and other parts of the channel. The c.2984 A > G (p.(N995S)) variant may alter the overall molecular conformation of the BK channel, weakening the closed state, or stabilizing the open state of the channel to make it open more frequently and for a longer period of time (Fig. 4).

In the era of precision medicine, the data in our study clearly demonstrate the critical importance of functional characterization of genomic variants of unknown significance identified by whole-genome sequencing or wholeexome sequencing. In this study, we identified four genomic variants in the BK channel in patients with epilepsy. Yet, c.2984 A > G (p.(N995S)) is a functional variant that causes epilepsy, while variants c.1554 G > T (p.(K518N)), c.1967A $>\mathrm{C}$ (p.(E656A)), and c.3476 A > G (p.(N1159S)) are not likely to cause epilepsy because of their lack of effects on BK channel function. Therefore, electrophysiological characterization of BK channel variants is required for distinguishing whether genomic variants have the potential to cause predisposition to a disease or not. It is interesting to note that other single-nucleotide polymorphisms were found within regions of three amino acids upstream or downstream of variants c.1554G $>\mathrm{T}$ (p.(K518N)), c.1967A $>\mathrm{C}$ (p. (E656A)), and c.3476 A > G (p.(N1159S)) (Supplementary Figure S4), but not of the c.2984 A > G (p.(N995S)) variant with a functional effect on the BK channel. This may suggest that the genomic regions at variants c. $1554 \mathrm{G}>\mathrm{T}$ (p. $(\mathrm{K} 518 \mathrm{~N})), c .1967 \mathrm{~A}>\mathrm{C}(\mathrm{p} .(\mathrm{E} 656 \mathrm{~A}))$, and c.3476 A > G (p. $(\mathrm{N} 1159 \mathrm{~S})$ ) are more tolerant for variants than the region at the c.2984 A > G (p.(N995S)) variant.

There is one limitation in our study. Our patch-clamping studies were carried out at room temperatures of $22-25^{\circ} \mathrm{C}$; therefore, we cannot exclude the possibility that variants c. $1554 \mathrm{G}>\mathrm{T} \quad(\mathrm{p} .(\mathrm{K} 518 \mathrm{~N})), \quad$ c.1967A $>\mathrm{C}$ (p.(E656A)), and c.3476 A > G (p.(N1159S)) may have an effect on the BK channel at a more physiological temperature of $37^{\circ} \mathrm{C}$.

In summary, our data demonstrate that variants in the BK channel $\alpha$-subunit cause epilepsy. Our study identifies the first de novo variant in the BK channel $\alpha$-subunit causing epilepsy. We have shown that the molecular mechanism for BK channel-associated epilepsy involves the allosteric voltage gating, but not the calcium-dependent activation of the channel. We further show that electrophysiological characterization is required to determine whether a BK channel variant is causative to disease. We suggest that the BK channel is a novel potential target for the development of treatments for epilepsy.

Acknowledgements We are grateful to Dr. Johannes Lemke for help and assistance and Dr. Jiuping Ding for the gift of the expression construct for KCNMA1 and KCNMB4. We thank Chengcheng Tan and Hongbo Xiong for help and assistance. This work was supported by the China National Natural Science Foundation grants (31430047, 81630002, and 91439129), 2016 Top-Notch Innovative Talent Development Project from the Bureau of Human Resources, and Social Security of Wuhan City, Chinese National Basic Research Programs (973 Programs 2013CB531101), Hubei Province Natural Science Key Program (2014CFA074), and NIH/NHLBI grants R01 HL121358 and R01 HL126729.

Conflict of interest The authors declare that they have no conflict of interest.

\section{References}

1. Orsini A, Zara F, Striano P. Recent advances in epilepsy genetics. Neurosci Lett. 2017;pii: S0304-3940(17)30402-0.

2. Sands TT, Choi H. Genetic testing in pediatric epilepsy. Curr Neurol Neurosci Rep. 2017;17:45.

3. Du W, Bautista JF, Yang H, et al. Calcium-sensitive potassium channelopathy in human epilepsy and paroxysmal movement disorder. Nat Genet. 2005;37:733-8.

4. Wang $\mathrm{C}$, Wu M, Qian J, et al. Identification of rare variants in TNNI3 with atrial fibrillation in a Chinese GeneID population. Mol Genet Genome. 2016;291:79-92.

5. Xu C, Yang Q, Xiong H, et al. Candidate pathway-based genomewide association studies identify novel associations of genomic variants in the complement system associated with coronary artery disease. Circ Cardiovasc Genet. 2014;7:887-94.

6. Wang $\mathrm{P}$, Yang $\mathrm{Q}, \mathrm{Wu} \mathrm{X}$, et al. Functional dominant-negative mutation of sodium channel subunit gene SCN3B associated with atrial fibrillation in a Chinese GeneID population. Biochem Biophys Res Commun. 2010;398:98-104.

7. Huang Y, Wang Z, Liu Y, et al. alphaB-Crystallin Interacts with Nav1.5 and Regulates Ubiquitination and Internalization of Cell Surface Nav1.5. J Biol Chem. 2016;291:11030-41.

8. Hou P, Zeng W, Gan G, et al. Inter-alpha/beta subunits coupling mediating pre-inactivation and augmented activation of $\mathrm{BKCa}$ (beta2). Sci Rep. 2013;3:1666.

9. Cui J, Aldrich RW. Allosteric linkage between voltage and $\mathrm{Ca}(2$ + )-dependent activation of BK-type mslo1 $\mathrm{K}(+)$ channels. Biochemistry. 2000;39:15612-9.

10. Wu L, Yong SL, Fan C, et al. Identification of a new co-factor, MOG1, required for the full function of cardiac sodium channel Nav 1.5. J Biol Chem. 2008;283:6968-78. 
11. Yang J, Krishnamoorthy G, Saxena A, et al. An epilepsy/dyskinesia-associated mutation enhances BK channel activation by potentiating $\mathrm{Ca} 2+$ sensing. Neuron. 2010;66:871-83.

12. Zhou Y, Lingle CJ. Paxilline inhibits BK channels by an almost exclusively closed-channel block mechanism. J General Physiol. 2014;144:415-40.

13. Brenner R, Chen QH, Vilaythong A, Toney GM, Noebels JL, Aldrich RW. BK channel beta4 subunit reduces dentate gyrus excitability and protects against temporal lobe seizures. Nat Neurosci. 2005;8:1752-9.

14. Meera P, Wallner M, Toro L. A neuronal beta subunit (KCNMB4) makes the large conductance, voltage- and $\mathrm{Ca} 2+$-activated $\mathrm{K}+$ channel resistant to charybdotoxin and iberiotoxin. Proc Natl Acad Sci USA. 2000;97:5562-7.

15. Wolff M, Johannesen KM, Hedrich U, et al. Genetic and phenotypic heterogeneity suggest therapeutic implications in SCN2Arelated disorders. Brain. 2017;140:1316-36.

16. Vu V, Verster AJ, Schertzberg M, et al. Natural variation in gene expression modulates the severity of mutant phenotypes. Cell. 2015;162:391-402.
17. Lee CY, Chen CC, Liou HH. Levetiracetam inhibits glutamate transmission through presynaptic P/Q-type calcium channels on the granule cells of the dentate gyrus. $\mathrm{Br} \mathrm{J}$ Pharmacol. 2009; 158:1753-62.

18. Nagarkatti N, Deshpande LS, DeLorenzo RJ. Levetiracetam inhibits both ryanodine and IP3 receptor activated calcium induced calcium release in hippocampal neurons in culture. Neurosci Lett. 2008;436:289-93.

19. Niespodziany I, Klitgaard H, Margineanu DG. Levetiracetam inhibits the high-voltage-activated $\mathrm{Ca}(2+)$ current in pyramidal neurones of rat hippocampal slices. Neurosci Lett. 2001;306: $5-8$.

20. Sheehan JJ, Benedetti BL, Barth AL. Anticonvulsant effects of the BK-channel antagonist paxilline. Epilepsia. 2009;50:711-20.

21. Wu Y, Yang Y, Ye S, Jiang Y. Structure of the gating ring from the human large-conductance $\mathrm{Ca}(2+)$-gated $\mathrm{K}(+)$ channel. Nature. 2010;466:393-7.

22. Yuan P, Leonetti MD, Pico AR, Hsiung Y, MacKinnon R. Structure of the human BK channel $\mathrm{Ca} 2+$-activation apparatus at 3.0 A resolution. Science. 2010;329:182-6.

\section{Affiliations}

\section{Xia $\mathrm{Li}^{1} \cdot$ Sibylle Poschmann ${ }^{2} \cdot$ Qiuyun $\mathrm{Chen}^{3,4} \cdot$ Walid Fazeli $^{5} \cdot$ Nelly Jouayed Oundjian ${ }^{6}$. Francesca M. Snoeijen-Schouwenaars ${ }^{7} \cdot$ Oliver Fricke $^{8} \cdot$ Erik-Jan Kamsteeg $^{9} \cdot$ Marjolein Willemsen $^{9,10}$. Qing Kenneth Wang ${ }^{1,3,4}$}

1 Laboratory of Molecular Biophysics of the Ministry of Education, College of Life Science and Technology, Center for Human Genome Research, Cardio-X Institute, Huazhong University of Science and Technology, Wuhan, Hubei Province, P. R. China

2 Children Hospital Dritter Orden, München, Germany

3 Center for Cardiovascular Genetics, Department of Molecular Cardiology, Lerner Research Institute, Department of Cardiovascular Medicine, Cleveland Clinic, OH, USA

4 Department of Genetics and Genome Science, Case Western Reserve University School of Medicine, Cleveland, OH, USA

5 University Children's Hospital Cologne, Pediatric Neurology, Cologne, Germany
6 Department of Pediatrics, Columbia University Medical Center, New York, NY, USA

7 Academic Department of Residential Care, Centre for Epileptology Kempenhaeghe, Heeze, The Netherlands

8 Department of Child and Adolescent Psychiatry and Neuropediatrics, Gemeinschaftskrankenhaus Herdecke, Witten/ Herdecke, Germany

9 Department of Human Genetics, Radboud University Medical Center, HB Nijmegen, The Netherlands

10 Department of Clinical Genetics, Maastricht University Medical Center, Maastricht, The Netherlands 